monitored by laser-doppler, and pro-inflammatory and oxidative stress markers were quantified by flow cytometry and RT-PCR. In a parallel cohort of SLE patients $(n=12)$, the effects of in vivo treatment with ubiquinol on spliceosome components was evaluated.

Results: As a general feature, a significant reduction in splicing factors and spliceosome components was found in all the leukocytes of SLE patients. Interestingly, we found a specific altered profile of splicing factors and spliceosome components when compared monocytes (U2AF1, FBP11, SRSF9), lymphocytes (RBM22, PRP8, SRSF5) and neutrophils (RNU4, CA150). The reduced levels of some components of spliceosome in both monocytes and neutrophils were linked to the occurrence of thrombotic events, foetal loss and arterial hypertension. In lymphocytes, those reduced levels were strongly related to the positivity for anti-dsDNA antibodies in SLE patients, thus suggesting that reduced spliceosome machinery would contribute to increase in altered autoantigen assembly, inducing increased autoantibody production. Correlation studies demonstrated an inverse relationship among reduced levels of spliceosome components/splicing factors and high activity of the disease (measured as SLEDAI), endothelial dysfunction, and increased expression levels of peroxides and peroxynitrites, as well as of altered mitochondrial membrane potential in monocytes and neutrophils. In vitro treatment of leukocytes from HDs with anti-dsDNA promoted a reduction in spliceosome components associated with the expression of proinflammatory and oxidative mediators. Finally, in vivo treatment with ubiquinol reversed reduced expression in SLE of spliceosome components related to their proaterothrombotic profile.

Conclusions: These results reveal the existence of SLE- associated spliceosome alterations -promoted by anti-dsDNA antibodies-which could be related to the development and activity of this autoimmune condition and have influence on the induction of mechanisms that drive atherothrombosis as well as the therapeutic response.

Acknowledgements: Funded by CTS7940 and ISCIII (PI15/01333 and RIER RD16/0012/0015).

Disclosure of Interest: None declared

DOI: 10.1136/annrheumdis-2017-eular.5036

\section{AB0129 HMGB1+ MICROPARTICLES IN SYSTEMIC LUPUS ERYTHEMATOSUS PATIENTS WITH LUPUS NEPHRITIS}

C. Burbano $^{1}$, J.A. Gómez-Puerta ${ }^{1}$, M. Rojas ${ }^{1}$, G. Vasquez ${ }^{1}$, J. Orejuela ${ }^{1}$, C.H. Muñoz ${ }^{2}$, A. Vanegas-García ${ }^{2}$, D. Castaño ${ }^{1}$. ${ }^{1}$ Universidad de Antioquia; ${ }^{2}$ Hospital Universitario de San Vicente Fundación, Medellín, Colombia

Background: High mobility group box protein 1 (HMGB1) is a nuclear DNAbinding protein that can function as an alarmin when is released from activated and dying cells. In association with nucleosomes, HMGB1 may contribute to the pathogenesis of systemic lupus erythematosus (SLE). Some previous reports have associated HMGB1 with the pathogenesis of cutaneous lupus and lupus nephritis (LN). HMGB1 may also be contained in microparticles (MPs). These vesicles have a wide spectrum of biological activities in intercellular communication, and they compete with apoptotic cells to bind mononuclear phagocytes.

Objectives: To evaluate the association of MP-HMGB1+ circulating with LN and to correlate them with LN activity.

Methods: Blood samples from 60 SLE patients were used to isolated MPs from platelet-poor plasma by centrifugation and their count, cell source and phenotype were characterized by flow cytometry. Renal pathology was reported using the standardized International Society of Nephrology/Renal Pathological Society classification. Inactive lupus nephritis (LN) was defined by the presence of one or more of the following criteria: $24 \mathrm{hrs}$ proteinuria $500 \mathrm{mg} / \mathrm{dl}$ or inactive urine sediments ( $<5$ red cells/HPF) and no red cell casts and no leucocyturia $(<5$ white cells/HPF) and stable serum creatinine.

Results: Mean age of SLE patients was $31.9 \pm 10.8$ years, and mean disease duration was $7.8 \pm 6.2$ years. $73 \%$ patients had $L N$ and $89 \%$ were female. Patients with LN had significantly higher frequency of MP-HMGB1+, no significant differences were found among patients with active versus inactive $L N$ or among patients with proliferative vs non-proliferative LN; MP-HMGB1+ had a moderate positive correlation with disease activity (SLEDAI, $r=0.367, p=0.020$ ), anti-C1q antibodies titers $(r=0.42, p=001)$ and 24 hours proteinuria $(r=0,33, p=, 032)$, but no correlation was found with activity or chronicity indexes on renal biopsies. A ROC curve for MP-HMGB1 + and renal involvement showed a good discriminative ability (AUC 0.706 ). A cutoff of $15.7 \%$ of MP-HMGB1+ showed the best discrimination threshold with a sensitivity of $63.3 \%$ and specificity of $83.3 \%$.

Conclusions: In our cohort of patients with SLE, MP-HMGB1+ was significantly higher in patients with $L N$ and in patients with active disease. Given the multiple implication of HMGB1 in SLE, including the active kidney recruitment of mononuclear phagocytes, we consider that MP-HMGB1+ could be considered as a potential biomarker for LN in SLE patients.

\section{References:}

[1] Harris, H. E. et al. HMGB1: A multifunctional alarmin driving autoimmune and inflammatory disease. Nat Rev Rheumatol. 8, 195-202 (2012).

[2] Zickert, A. et al. Renal expression and serum levels of high mobility group box 1 protein in lupus nephritis. Arthritis Res Ther. 20;14(1):R36 (2012).

Acknowledgements: C. Burbano is recipient of a doctoral scholarship from COLCIENCIAS (call 617-2013). The authors are grateful to the grants "Sostenibilidad and Sistema Universitario de Investigaciones, CODI (2013-05-42869836) from Universidad de Antioquia, and to COLCIENCIAS (111565740575)".

Disclosure of Interest: None declared

DOI: 10.1136/annrheumdis-2017-eular.4397

\section{AB0130 MESENCHYMAL STEM CELLS ALLEVIATE SLE THROUGH PROMOTING TREG CELLS BY HLA-G}

C. Chen, J. Liang, G. Yao, Z. Zhang, S. Huang, L. Sun. The Affiliated Drum Tower Hospital of Nanjing University Medical School, Nanjing, China

Background: Soluble human leukocyte antigen-G (sHLA-G) is a non-classical HLA class I molecule, exhibiting strong immunosuppressive properties by inducing the differentiation of T regulatory cells (Treg). Mesenchymal stem cells (MSCs) transplantation alleviates disease progression in systemic lupus erythematosus (SLE) patients. However, the underlying mechanisms are largely unknown.

Objectives: The aim of the present study is to explore whether SHLA-G is involved in upregulating Treg cells by MSCs, which contributes to therapeutic effects of MSCs transplantation in SLE.

Methods: The serum SHLA-G levels of SLE patients and healthy controls were detected by ELISA. The percentages of peripheral blood CD4 + ILT2 +, CD8 + ILT2 +, CD19 + ILT2 + cells and Treg cells were determined by flow cytometry. Ten patients with active SLE, refractory to conventional therapies, were infused with MSCs and serum SHLA-G was measured $24 \mathrm{~h}$ after infusion. Peripheral blood mononuclear cells (PBMCs) were isolated from SLE patients and co-cultured with UC-MSCs for 3 days at different ratios $(50: 1,10: 1$, and 2:1) with or without anti-HLA-G antibodies, and the frequencies of CD4 + CD25 + Foxp3 + T cells were then determined by flow cytometry.

Results: The concentrations of serum SHLA-G were comparable between SLE patients and healthy controls. However, there was a negative correlation between sHLA-G levels and SLE disease activity index (SLEDAI) scores in active SLE patients (SLEDAI >4). We found that serum SHLA-G levels were negatively correlated with blood urea nitrogen, serum creatinine and 24-hour urine protein in SLE patients. The sHLA-G levels were significantly lower in SLE patients with renal involvement than those without renal involvement. The expression of ILT2 on CD4 + T cells from SLE patients decreased significantly compared to that of healthy controls. A positive correlation between the frequencies of Treg and CD4 + ILT2 + T cells was found in SLE patients. The levels of SHLA-G increased $24 \mathrm{~h}$ after UC-MSC transplantation. The frequencies of Treg cells and the expressions of ILT2 on CD4 + Tcells were significantly increased $24 \mathrm{~h}$ after transplantations. In vitro studies showed that MSCs increased the frequency of Treg cells in SLE patients in a dose-dependent manner, which was partly abrogated by the anti-HLA-G antibody.

Conclusions: Our results suggested that MSCs might alleviate SLE through upregulating Treg cells, which was partly dependent on SHLA-G.

Disclosure of Interest: None declared

DOI: 10.1136/annrheumdis-2017-eular.3600

\section{AB0131 PLATELET MICROPARTICLES (PMPS) ARE HIGHER IN PATIENTS WITH SLE COMPARED TO HEALTHY CONTROLS}

C.-L.M. Murphy, V. Ripoll-Nunez, C. Periculeous, T. McDonnell, S. Croca,

I. Giles, A. Rahman. Rheumatology, University College London Hospital, London, United Kingdom

Background: Patients with SLE have an increased risk of cardiovascular disease (CVD) and reasons for this are unknown. Microparticles (MPs) are membrane-bound particles released by many cells, 70-90\% are derived from platelets. MPs are a rich source of autoantigens, including DNA and have many functions including thrombosis and inflammation. PMPs can induce foam cell formation and promote adherence of platelets to endothelial lesions. MPs are potentially important in the pathogenesis of autoimmune rheumatic disease and in cardiovascular disease.

Objectives: To determine if EMPs and PMPs are increased in patients with SLE. Our secondary aim was to determine if there was a difference between those with/without subclinical cardiovascular disease and healthy controls.

Methods: Atherosclerotic plaque was previously determined in patients with SLE who had no known history of CVD by carotid and femoral ultrasound scans. Plaque and thickened intima media thickness $(\mathrm{IMT})>0.1 \mathrm{~cm}$ were defined according to the Mannheim Carotid Consensus. Data regarding plaque area, IMT and echolucency were collected. Plasma was stored at $-80^{\circ}$ at the time of scan. Plasma from $n=57$; plaque $(n=16)$, no plaque $(n=23)$ and healthy controls $(H C)(n=18)$ were analysed for presence of endothelial and platelet microparticles (EMPs and PMPs), as per protocol. Samples were stained with Annexin V and platelet and endothelial antibodies, CD42a, CD31, CD105 and CD144. MPs were measured using flow cytometry. Statistical analysis was carried out using PRISM. Results: A total of 57 plasma samples were tested. The average age of those with SLE was $45 \pm 12$ years. $96 \%$ were female. $56 \%$ were Caucasian, $18 \%$ Asian, $21 \%$ Afrocaribbean, $5 \%$ other ethnicity. $18 \%$ of the SLE patients were smokers. Of the $18 \mathrm{HC}$, the average age was $37 \pm 7$ years, $83 \%$ were female. $88 \%$ were Caucasian and $12 \%$ other ethnicity. $11 \%$ of the HCs were smokers. PMPs were significantly higher in patients with SLE compared to healthy controls $(p=0.025)$. Patients with SLE without plaque had more PMPs compared to healthy controls 